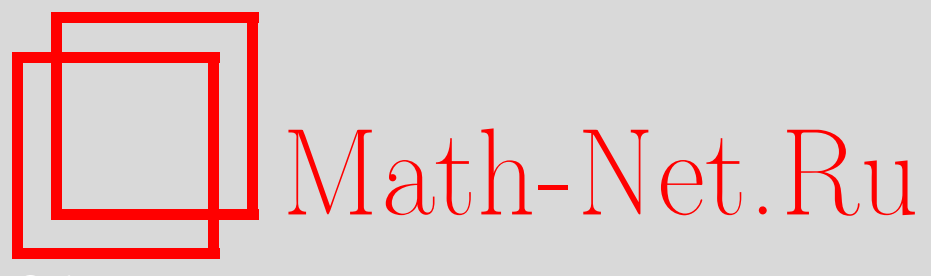

А. Е. Мамонтов, Существование глобальных решений многомерных уравнений сжимаемой жидкости Бингама, Матем. заметки, 2007, том 82, выпуск 4, 560-577

DOI: https://doi.org/10.4213/mzm3825

Использование Общероссийского математического портала Math-Net.Ru подразумевает, что вы прочитали и согласны с пользовательским соглашением http://www.mathnet.ru/rus/agreement

Параметры загрузки:

IP : 3.82 .47 .9

26 апреля 2023 г., 12:42:16

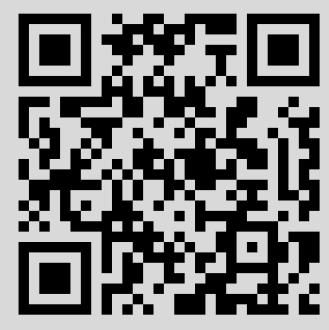


Том 82 выпуск 4 октябрь 2007

УДК 517.958

\section{Существование глобальных решений многомерных уравнений сжимаемой жидкости Бингама}

\section{А. Е. Мамонтов}

Рассматриваются уравнения, описывающие многомерные движения сжимаемых вязких (неньютоновских) жидкостей типа Бингама, т.е. с многозначной функцией, связывающей напряжения с тензором скоростей деформаций. Доказана теорема существования в целом по времени и начальным данным для первой начально-краевой задачи, соответствующей течениям в ограниченной области, в классе "слабых" обобщенных решений. При этом допускается анизотропная связь между тензорами напряжений и скоростей деформаций и подробно исследуются допустимые связи такого рода.

Библиография: 15 названий.

Введение. Будем рассматривать систему уравнений, описывающих течения вязких (в том числе неньютоновских) сжимаемых жидкостей

$$
\begin{aligned}
\frac{\partial \rho}{\partial t}+\operatorname{div}(\rho \mathbf{u}) & =0 \\
\frac{\partial(\rho \mathbf{u})}{\partial t}+\operatorname{div}(\rho \mathbf{u} \otimes \mathbf{u}) & =\operatorname{div} \mathbb{P}_{r}+\rho \mathbf{f},
\end{aligned}
$$

где $\rho$ - плотность жидкости (неотрицательная функция), $\mathbf{u}-$ вектор скорости, $\mathbf{f}-$ заданный вектор внешних массовых сил, $\mathbb{P}_{r}$ - тензор напряжений, div - оператор дивергенции по пространственным переменным $\mathbf{x} \in \mathbb{R}^{n}$, a $t$ - время. Жидкость Бингама [1], [2] характеризуется тем, что в ней при малых напряжениях тензор скоростей деформаций $\mathbb{D}(\mathbf{u})=\operatorname{Sym}(\nabla \otimes \mathbf{u})$ равен 0 (далее для поля $\mathbf{u}$ будем вместо $\mathbb{D}(\mathbf{u})$ писать просто $\mathbb{D})$, т.е. в областях с малыми $\mathbb{P}_{r}$ (за вычетом шаровой части) движение твердотельное (строго говоря, такие среды не являются жидкостями в смысле аксиом (постулатов) Стокса [3]). Модели жидкостей Бингама находят свое применение при изучении движений таких сред, как пасты, цементы, суспензии, некоторые виды нефтей и др. [4], [5].

Таким образом, жидкость Бингама описывается определяющим уравнением (замыкающим систему $(0.1),(0.2))$ вида

$$
\mathbb{P}_{r}=\mathbb{P}_{f}+\mathbb{P}_{b},
$$

Работа выполнена при поддержке Российского фонда фундаментальных исследований (грант № 07-01-00550). 
где $\mathbb{P}_{f}-$ тензор напряжений стоксовой (т.е. удовлетворяющей аксиомам Стокса) жидкости, а $\mathbb{P}_{b}$ есть многозначная функция от $\mathbb{D}$, задаваемая формулой

$$
\mathbb{P}_{b}=p_{*} \begin{cases}\mathbb{T}\left(\frac{\mathbb{D}}{|\mathbb{D}|}\right), & \mathbb{D} \neq 0, \\ \text { любой из } \overline{\mathscr{P}}, & \mathbb{D}=0 .\end{cases}
$$

Здесь $\mathscr{P}$ - некоторая ограниченная выпуклая область в пространстве $\mathbb{S}_{n}$ симметричных тензоров ранга 2 , действующих в $\mathbb{R}^{n}$, причем $0 \in \mathscr{P}, \rho(0, \partial \mathscr{P})=1 ; p_{*} \geqslant 0-$ заданное (постоянное) пороговое напряжение, а $\mathbb{T}$ - тензорное поле, действующее из единичной сферы $S_{1} \subset \mathbb{S}_{n}$ в $\partial \mathscr{P}$. Другими словами, $p_{*} \mathscr{P}$ есть область напряжений, соответствующих твердотельному движению (так как $\mathbb{P}_{b} \in p_{*} \overline{\mathscr{P}}$, а $\mathbb{P}_{f}$ стоксов), a $\mathbb{T}$ определяет связь напряжений и скоростей деформаций при выходе напряжений в критическую зону $p_{*} \partial \mathscr{P}$. Подробнее связь $(0.4)$ будет изучена в пп. 1,2 . В $(0.4)$ и далее модуль тензора $|\mathbb{B}|=(\mathbb{B}: \mathbb{B})^{1 / 2}$. Отметим, что значение $p_{*}=0$ соответствует $\mathbb{P}_{b} \equiv 0$, т.е. стоксовой жидкости.

В настоящей работе общий анизотропный случай (0.4) рассмотрен в связи с тем, что это не требует серьезных дополнительных усилий по сравнению с изотропным случаем (когда $\mathscr{P}$ есть единичный шар в $\mathbb{S}_{n}$, а $\mathbb{T}$ - тождественное отображение), обычно рассматриваемым в литературе. Вопрос о разрешимости в целом по времени задач для системы (0.1)-(0.4) до сих пор рассматривался (при $p_{*}>0$ ) только для случая одномерных движений $(n=1)$ или для несжимаемой жидкости. За подробностями мы отсылаем читателя к работам [6]-[8] и библиографиям в них.

В настоящей статье будет доказано существование в целом решений первой начально-краевой задачи (задачи А) для системы (0.1)-(0.4) (см. постановку задачи А в п. 1). При этом мы будем опираться на результат [9], [10], полученный для этой задачи в случае $p_{*}=0$. Указанный результат неприменим непосредственно к случаю $p_{*}>0$, так как тогда (0.3) определяет многозначную функцию от $\mathbb{D}$ (см. также замечание 1.4). Таким образом, имеется два пути распространения результата [9], [10] на интересующий нас случай $p_{*}>0$ :

1) обосновать возможность повторения результатов [9], [10] в этом случае, проведя заново все доказательства в изменившейся ситуации;

2) регуляризовав бингамовский тензор $\mathbb{P}_{b}$ и сведя его к стоксовому, перейти к пределу в построенных на основе [9], [10] решениях регуляризованной задачи (аналогично тому, как это делалось в [6] для случая $n=1$ ).

Второй путь предпочтительнее, поскольку на нем удобно отследить появление “твердотельных зон” (где $\mathbb{D}=0$ ) и поведение в них напряжений. Этот путь мы и изберем в настоящей работе.

Основной результат статьи сформулирован в теореме 3.2. Его доказательству посвящен п. 3. В пп. 1, 2 приводятся постановка основной задачи, вспомогательные построения и примеры; в п. 4 собраны несколько технических утверждений о выпуклых функционалах.

1. Постановка задачи А. Будем рассматривать начально-краевую задачу для системы (0.1)-(0.4), описывающую движение в ограниченной области $\Omega \subset \mathbb{R}^{n}$ с гладкой границей. Точнее, речь идет о первой начально-краевой задаче в цилиндре $Q_{T}=\Omega \times(0, T)$ (с условиями прилипания на $\left.\partial \Omega \times(0, T)\right)$ :

$$
\left.\rho\right|_{t=0}=\rho_{0} \geqslant 0,\left.\quad \rho \mathbf{u}\right|_{t=0}=\mathbf{w}_{0},\left.\quad \mathbf{u}\right|_{\partial \Omega}=0 .
$$


Начальные данные ставятся для импульса $\rho \mathbf{u}$, а не для скорости $\mathbf{u}$, так как вообще говоря, только импульс принимает свои начальные значения в рассматриваемом нами классе слабых решений, в котором возможно $\rho=0$ на множестве ненулевой меры, в то время как $\rho$ играет роль веса при $\partial \mathbf{u} / \partial t$ в $(0.2)$. Налагая естественное с физической точки зрения ограничение $\operatorname{mes}\left\{\mathbf{w}_{0} \neq 0, \rho_{0}=0\right\}=0$, под значением $\left.\mathbf{u}\right|_{t=0}$ будем понимать отношение $\mathbf{w}_{0} / \rho_{0}$, произвольным образом продолженное на множество $\left\{\rho_{0}=0\right\}$.

Следуя [9], [10], будем задавать стоксову часть $\mathbb{P}_{f}$ тензора $\mathbb{P}_{r}$ в виде

$$
\mathbb{P}_{f}=-\rho \mathbb{I}+\mathbb{P}(\mathbf{u})
$$

(т.е. давление $p(\rho)=\rho$ ), где $\mathbb{P}$ является оператором произвольной природы (возможно, и нелокальным), удовлетворяющим лишь следующим аксиомам:

А1) $\mathbb{P}$ коэрцитивен, т.е.

$$
L(\mathbf{u}) \equiv \int_{\Omega} \mathbb{P}(\mathbf{u}): \mathbb{D}(\mathbf{u}) d \mathbf{x} \geqslant \int_{\Omega} M(|\mathbb{D}(\mathbf{u})|) d \mathbf{x} \quad \text { при всех } \quad \mathbf{u} \in X ;
$$

А2) $\mathbb{P}$ монотонен, т.е.

$$
\int_{\Omega}(\mathbb{P}(\mathbf{u})-\mathbb{P}(\mathbf{v})): \mathbb{D}(\mathbf{u}-\mathbf{v}) d \mathbf{x} \geqslant 0 \quad \text { при всех } \quad \mathbf{u}, \mathbf{v} \in X ;
$$

А3) $\mathbb{P}(\cdot)$ действует в определенном смысле ограниченным образом:

$$
\int_{\Omega} \bar{M}(|\mathbb{P}(\mathbf{u})|) d \mathbf{x} \leqslant C\left(1+\int_{\Omega} M(|\mathbb{D}(\mathbf{u})|) d \mathbf{x}\right) \quad \text { для всех } \quad \mathbf{u} \in X ;
$$

также $\mathbb{P}$ непрерывен в следующем смысле:

А4) $\mathbb{P}(\mathbf{u}-\varepsilon \mathbf{v}) \rightarrow \mathbb{P}(\mathbf{u}) *$-слабо в $L_{\bar{M}}(\Omega)$ при $\varepsilon \rightarrow 0$ для всех $\mathbf{u}, \mathbf{v} \in X$;

А5) если $\mathbb{D}\left(\mathbf{u}_{\varepsilon}\right) \rightarrow \mathbb{D}(\mathbf{u})$ п.в. в $Q_{T}$ и $\mathbb{D}\left(\mathbf{u}_{\varepsilon}\right)$ ограничены в $K_{M}\left(Q_{T}\right)$, то $\mathbb{P}\left(\mathbf{u}_{\varepsilon}\right) \rightarrow \mathbb{P}(\mathbf{u})$ в $\mathscr{D}^{\prime}\left(Q_{T}\right)$;

и, наконец, имеют место следующие свойства (типа выпуклости):

A6) интеграл $\int_{0}^{t} L(\mathbf{u}) d s$ задает функционал от $\mathbf{u}, *$-слабо полунепрерывный снизу, т.е. если $\mathbb{D}\left(\mathbf{u}_{\varepsilon}\right) \rightarrow \mathbb{D}(\mathbf{u}) *$-слабо в $L_{M}\left(Q_{t}\right)$, то

$$
\int_{0}^{t} L(\mathbf{u}) d s \leqslant \underline{\lim } \int_{0}^{t} L\left(\mathbf{u}_{\varepsilon}\right) d s
$$

А7) если $\mathbb{D}\left(\mathbf{u}_{\varepsilon}\right) \rightarrow \mathbb{D}(\mathbf{u})$ *-слабо в $L_{M}\left(Q_{t}\right)$, и

$$
\int_{0}^{t} L(\mathbf{u}) d s \geqslant \varlimsup \int_{0}^{t} L\left(\mathbf{u}_{\varepsilon}\right) d s
$$

то для некоторой последовательности $\mathbb{D}\left(\mathbf{u}_{\varepsilon_{k}}\right) \rightarrow \mathbb{D}(\mathbf{u})$ п.в. в $Q_{t}$.

Здесь $M$ означает N-функцию, обладающую свойством

$$
M(s) \geqslant \exp (s), \quad s \gg 1
$$

$\bar{M}$ - функция, дополнительная к ней;

$$
X=\left\{\mathbf{u}\left|\mathbb{D}(\mathbf{u}) \in L_{M}(\Omega), \mathbf{u}\right|_{\partial \Omega}=0\right\} ; \quad\|\mathbf{u}\|_{X}=\|\mathbb{D}(\mathbf{u})\|_{L_{M}(\Omega)} .
$$


Теория $\mathrm{N}$-функций, пространств $L_{M}$ и классов $K_{M}$ Орлича изложена в [11] (с той оговоркой, что там вместо $L_{M}, K_{M}$ обозначено соответственно $\left.L_{M}^{*}, L_{M}\right)$. Свойства пространства $X$ описаны в [9].

Теорема существования в целом для задачи $(0.1)-(0.4),(1.1),(1.2)$ в случае $p_{*}=0$ получена в [9], [10] в рамках аксиом А1-А4 и ограничения (1.3). При этом было показано, что что в рамках принятого подхода к оценкам решения задачи требование (1.3) не может быть существенно ослаблено. В настоящей работе мы не заботимся об оптимальности требований на связь $\mathbb{P}_{r}$ и $\mathbb{D}$ при больших $|\mathbb{D}|$ (т.е. на рост функции $M)$, так же как и об общности $p(\rho)$, поскольку наша основная цель отследить специфику бингамовской жидкости (т.е. малые $|\mathbb{D}|)$.

В качестве примера тензоров $\mathbb{P}$, удовлетворяющих аксиомам A1-A7, можно указать следующий класс.

Пример 1.1. Рассмотрим потенциальные тензоры - градиенты $\mathbb{P}=\partial V / \partial \mathbb{D}$ потенциалов вида

$$
V=\Lambda\left((\operatorname{tr} \mathbb{D})^{2}\right)+\sum_{s=1}^{N} \Gamma_{s}\left(\left|\mathbb{D}^{s}\right|^{2}\right)
$$

с произвольным $N \in \mathbb{N}$. Такого рода тензоры $\mathbb{P}$ подробно изучены в [12]. Аксиомы A1-А4 выполнены, если $\Lambda$ и все $\Gamma_{s}-$ неубывающие выпуклые функции класса $C^{1}$, причем $\Gamma_{1}$ растет существенно быстрее всех остальных функций, а $M(\xi)=2 \Gamma_{1}^{\prime}\left(\xi^{2}\right) \xi^{2}$ удовлетворяет $\Delta_{3}$-условию (фактически достаточно (1.3)). Аксиома А6 обеспечивается выпуклостью функций $\Lambda^{\prime}\left(\xi^{2}\right) \xi^{2}$ и $\Gamma_{s}^{\prime}\left(\xi^{2}\right) \xi^{2}$. В самом деле, (1.4) означает, что (cм. [12])

$$
\mathbb{P}(\mathbf{u})=2 \Lambda^{\prime}\left((\operatorname{tr} \mathbb{D})^{2}\right)(\operatorname{tr} \mathbb{D}) \cdot \mathbb{I}+\sum_{s=1}^{N} 2 s \Gamma_{s}^{\prime}\left(\left|\mathbb{D}^{s}\right|^{2}\right) \mathbb{D}^{2 s-1},
$$

и обоснование аксиомы А6 опирается на следствие 4.3. Для выполнения аксиомы А7 (см. пример 4.11) следует потребовать $\Lambda, \Gamma_{s} \in C^{3}$, а выпуклость функций $\Lambda^{\prime}\left(\xi^{2}\right) \xi^{2}$ и $\Gamma_{s}^{\prime}\left(\xi^{2}\right) \xi^{2}$ следует обеспечить строгую (точнее, удовлетворить оценки вида (4.3)). Наконец, аксиома А5 обеспечена в силу следующих соображений: если $\mathbb{D}\left(\mathbf{u}_{\varepsilon}\right)$ ограничены в $K_{M}\left(Q_{T}\right)$, то по аксиоме А3 это дает ограниченность $\mathbb{P}\left(\mathbf{u}_{\varepsilon}\right)$ в $K_{\bar{M}}\left(Q_{T}\right)$, а тогда $[11 ;$ с. 117,135$] \mathbb{P}\left(\mathbf{u}_{\varepsilon}\right)$ имеют равностепенно непрерывные нормы в любом $K_{N}\left(Q_{T}\right)$ с $N \prec \prec \bar{M}$, но тогда из $\mathbb{D}\left(\mathbf{u}_{\varepsilon}\right) \rightarrow \mathbb{D}(\mathbf{u})$ п.в. (а значит, и $\mathbb{P}\left(\mathbf{u}_{\varepsilon}\right) \rightarrow \mathbb{P}(\mathbf{u})$ п.в.) следует $\mathbb{P}\left(\mathbf{u}_{\varepsilon}\right) \rightarrow \mathbb{P}(\mathbf{u})$ сильно в $K_{N}\left(Q_{T}\right)$, что и требовалось.

Опишем теперь рассматриваемый нами класс бингамовских тензоров $\mathbb{P}_{b}$. Как сказано во введении, $\mathbb{P}_{b}$ будем брать в виде (0.4). При этом будем предполагать выполнение следующих аксиом:

B1) $\mathbb{T}$ - непрерывное отображение;

В2) $\mu(\mathbb{B}) \equiv \mathbb{T}(\mathbb{B}): \mathbb{B}>0$ при всех $\mathbb{B} \in S_{1}$;

В3) $\mathbb{T}\left(\mathbb{B}_{2}\right): \mathbb{B}_{1} \leqslant \mathbb{T}\left(\mathbb{B}_{1}\right): \mathbb{B}_{1}=\mu\left(\mathbb{B}_{1}\right)$ при всех $\mathbb{B}_{1,2} \in S_{1}$.

ЗАмечАниЕ 1.2. Из выписанных аксиом следует, что $\mu$-непрерывная функция со значениями из интервала вида $\left[\mu_{0}, 1\right]$, где $\mu_{0}=$ const $>0$.

Из аксиомы В3 вытекает свойство

В4) функционал

$$
\varkappa(\mathbb{D}) \equiv \mu\left(\frac{\mathbb{D}}{|\mathbb{D}|}\right)|\mathbb{D}|
$$

(доопределенный нулем при $\mathbb{D}=0$ ) выпуклый. 
В самом деле, для $\mathbb{D}_{\theta}=\theta \mathbb{D}_{1}+(1-\theta) \mathbb{D}_{2}$ имеем ввиду В3:

$$
\mathbb{T}\left(\frac{\mathbb{D}_{\theta}}{\left|\mathbb{D}_{\theta}\right|}\right): \mathbb{D}_{k} \leqslant \varkappa\left(\mathbb{D}_{k}\right), \quad k=1,2,
$$

откуда

$$
\varkappa\left(\mathbb{D}_{\theta}\right)=\mathbb{T}\left(\frac{\mathbb{D}_{\theta}}{\left|\mathbb{D}_{\theta}\right|}\right): \mathbb{D}_{\theta} \leqslant \theta \varkappa\left(\mathbb{D}_{1}\right)+(1-\theta) \varkappa\left(\mathbb{D}_{2}\right),
$$

что и требовалось. Отметим, что $\varkappa-$ непрерывный функционал.

Тривиальный пример описанного класса тензоров $\mathbb{P}_{b}$ дается упомянутым во введении случаем изотропной жидкости Бингама, когда $\mu \equiv 1, \varkappa(\mathbb{D})=|\mathbb{D}|$ и выполнение аксиом B1-B3 очевидно. При этом тензор $\mathbb{P}_{b}$ будет потенциальным, причем его потенциал $V_{b}(\mathbb{D})=p_{*}|\mathbb{D}|$ с той оговоркой, что при таком представлении при $\mathbb{D} \rightarrow 0$ $\mathbb{P}_{b}$ будет принимать значения только на сфере $p_{*} \partial \mathscr{P}=p_{*} S_{1}$ (однако при переходе к слабому пределу, как в п. 3, значения будут приниматься во всем шаре $p_{*} \overline{\mathscr{P}}$ ). Кроме того, описанные тензор и потенциал имеют вид (1.4), (1.5) с $\widetilde{\Gamma}_{1}(\xi)=p_{*} \sqrt{\xi}$ (остальные члены нулевые), так что если $\mathbb{P}$ также задается формулами (1.4), (1.5), то это представление имеет место и для результирующих потенциала $V_{r}=V_{b}+V$ и тензора $\mathbb{P}_{b}+\mathbb{P}$, но тогда $\widetilde{\Gamma}_{1}+\Gamma_{1}$ не может быть выпуклой в окрестности 0 , поскольку $\Gamma_{1}^{\prime} \geqslant 0$, откуда $\left(\widetilde{\Gamma}_{1}+\Gamma_{1}\right)^{\prime}(+0)=+\infty$. Поэтому $\mathbb{P}_{b}$ все же удобнее рассматривать как отдельное слагаемое.

Нетривиальные примеры пар $(\mathscr{P}, \mathbb{T})$ (т.е. анизотропных $\left.\mathbb{P}_{b}\right)$, удовлетворяющих аксиомам В1-В3, построены в п. 2 .

Теперь мы готовы окончательно сформулировать основную задачу.

ОПРЕДЕЛЕНИЕ 1.3. Задачей А назовем задачу (0.1)-(0.4), (1.1), (1.2), в которой тензор $\mathbb{P}$ обладает свойствами А1-A7 с ограничением (1.3), а поле $\mathbb{T}$ - свойствами В1-В3 (а значит, и В4).

Как сказано во введении, задача А будет решаться при помощи построения специальных аппроксимирующих задач. При этом нам потребуется обозначение

$$
\mathbb{P}_{b 0}=p_{*} \begin{cases}\mathbb{T}\left(\frac{\mathbb{D}}{|\mathbb{D}|}\right), & \mathbb{D} \neq 0, \\ 0, & \mathbb{D}=0,\end{cases}
$$

а также семейство регуляризованных тензоров $\mathbb{P}_{b \varepsilon}, \varepsilon>0$, обладающее следующими свойствами:

C1) $\mathbb{P}_{b \varepsilon} \in p_{*} \overline{\mathscr{P}}$ всюду;

$\mathrm{C} 2)$ при $\mathbb{D}=0$ верно $\mathbb{P}_{b \varepsilon}=0$ (т.е. это стоксов тензор);

C3) $\mathbb{P}_{b \varepsilon}(\mathbf{v}) \rightarrow \mathbb{P}_{b}(\mathbf{v})$ при $\varepsilon \rightarrow 0$ равномерно по $|\mathbb{D}(\mathbf{v})| \in\left[\delta, d_{0}\right]$ при всех $\delta>0$ с фиксированным $d_{0}>0$;

C4) $\mathbb{P}_{b \varepsilon}=\mathbb{P}_{b}$ при $|\mathbb{D}| \geqslant d_{0}$;

$\mathrm{C} 5)$ сумма $\left(\mathbb{P}+\mathbb{P}_{b \varepsilon}\right)$ удовлетворяет аксиомам $\mathrm{A} 1-\mathrm{A} 3$, а $\mathbb{P}_{b \varepsilon}-$ аксиомам $\mathrm{A} 2-\mathrm{A} 4$ (значит, А4 верно и для суммы).

Такое семейство, как показано в п. 2, всегда может быть построено (т.е. C1-C5 не являются дополнительными ограничениями), если только выполнены свойства В1-В3. Из свойств C1-C5 следуют также следующие свойства:

C6) $\mathbb{P}_{b \varepsilon} \rightarrow \mathbb{P}_{b 0}$ при всех $\mathbb{D}$ поточечно, и равномерно по $|\mathbb{D}| \in[\delta,+\infty)$ при всех $\delta>0$;

$\mathrm{C} 7)$ если $\mathbb{D}(\mathbf{v}) \neq 0$, то из $\mathbb{D}\left(\mathbf{v}_{\varepsilon}\right) \rightarrow \mathbb{D}(\mathbf{v})$ следует $\mathbb{P}_{b \varepsilon}\left(\mathbf{v}_{\varepsilon}\right) \rightarrow \mathbb{P}_{b}(\mathbf{v})$; 
С8) для любого семейства $\left\{\mathbf{v}_{\varepsilon}\right\}$ верна оценка

$$
\left|\left(\mathbb{P}_{b \varepsilon}\left(\mathbf{v}_{\varepsilon}\right)-\mathbb{P}_{b 0}\left(\mathbf{v}_{\varepsilon}\right)\right): \mathbb{D}\left(\mathbf{v}_{\varepsilon}\right)\right| \leqslant n(\varepsilon) \rightarrow 0 \quad \text { при } \quad \varepsilon \rightarrow 0,
$$

где $n(\varepsilon)$ не зависит от $\left\{\mathbf{v}_{\varepsilon}\right\}$.

ДокАЗАтЕльство. Свойство С6 очевидно. Далее, имеем оценку

$$
\left|\mathbb{P}_{b \varepsilon}\left(\mathbf{v}_{\varepsilon}\right)-\mathbb{P}_{b}(\mathbf{v})\right| \leqslant\left|\mathbb{P}_{b \varepsilon}\left(\mathbf{v}_{\varepsilon}\right)-\mathbb{P}_{b}\left(\mathbf{v}_{\varepsilon}\right)\right|+\left|\mathbb{P}_{b}\left(\mathbf{v}_{\varepsilon}\right)-\mathbb{P}_{b}(\mathbf{v})\right|
$$

из которой очевидно следует С7 в силу свойства С6 и тривиальной оценки $\left|\mathbb{D}\left(\mathbf{v}_{\varepsilon}\right)\right| \geqslant$ $\delta>0$. Наконец, на основании свойств $\mathrm{C} 1, \mathrm{C} 4$ левая часть (1.6) оценивается сверху величиной (с любым $\left.\varepsilon_{1}>0\right)$

$$
2 p_{*} \varepsilon_{1}+d_{0} \sup _{|\mathbb{D}(\mathbf{v})| \in\left[\varepsilon_{1}, d_{0}\right]}\left|\mathbb{P}_{b \varepsilon}(\mathbf{v})-\mathbb{P}_{b 0}(\mathbf{v})\right|,
$$

которая не зависит от $\left\{\mathbf{v}_{\varepsilon}\right\}$ и может быть в силу свойства С3 сделана сколь угодно малой при $\varepsilon \rightarrow 0$ при подходящем подборе $\varepsilon_{1}$.

ЗАмечание 1.4. Функционал $\mathbb{P}_{b}: \mathbb{D}=\mathbb{P}_{b 0}: \mathbb{D}=p_{*} \varkappa(\mathbb{D})$, заданный в $\mathbb{S}_{n}$, является функционалом класса (4.2), поэтому по следствию 4.3 функционал

$$
N(\mathbf{u}) \equiv \int_{0}^{t} \int_{\Omega}\left(\mathbb{P}(\mathbf{u})+\mathbb{P}_{b 0}(\mathbf{u})\right): \mathbb{D}(\mathbf{u}) d \mathbf{x} d s=\int_{0}^{t} L(\mathbf{u}) d s+p_{*} \int_{0}^{t} \int_{\Omega} \varkappa(\mathbb{D}(\mathbf{u})) d \mathbf{x} d s
$$

также удовлетворяет аксиоме А6. Как будет показано в п. 2 (для более общего тензора $\left.\mathbb{P}+\mathbb{P}_{b \varepsilon}\right)$, тензор $\mathbb{P}+\mathbb{P}_{b}$ удовлетворяет всем аксиомам $\mathrm{A} 1-\mathrm{A} 4$, т.е. он попадает в условия [9], [10], и лишь его неоднозначность, как сказано во введении, препятствует непосредственному применению результатов упомянутых работ.

2. Построение $\mathbb{P}_{b \varepsilon}$ и нетривиальных пар $(\mathscr{P}, \mathbb{T})$. Как было анонсировано в п. 1, построим пример семейства $\mathbb{P}_{b \varepsilon}$, обладающего свойствами C1-C5, опираясь лишь на аксиомы В1-В3 для поля $\mathbb{T}$ и $\mathrm{A} 1-\mathrm{A} 4$ для $\mathbb{P}$. Таким примером является

$$
\mathbb{P}_{b \varepsilon}=\varphi_{\varepsilon}(\varkappa(\mathbb{D})) \mathbb{P}_{b}
$$

где $\varphi_{\varepsilon}-$ произвольное семейство функций, заданных на $\mathbb{R}^{+}$и обладающих следующими свойствами:

D1) $\varphi_{\varepsilon}(0)=0, \varphi_{\varepsilon} \equiv 1$ на $\left[\mu_{0} d_{0},+\infty\right)$, где $d_{0}=$ const $>0$;

D2) $\varphi_{\varepsilon}(\cdot)$ непрерывны и не убывают;

D3) $\varphi_{\varepsilon} \rightarrow 1$ при $\varepsilon \rightarrow 0$ равномерно на $\left[\delta, d_{0}\right]$ при всех $\delta>0$.

$\mathrm{B}$ частности, из D1-D3 следует $0 \leqslant \varphi_{\varepsilon} \leqslant 1$ и непрерывность $\mathbb{P}_{b \varepsilon}$ как функции от $\mathbb{D}$. В качестве $\varphi_{\varepsilon}$ можно взять, например, функции

$$
\varphi_{\varepsilon}(z)= \begin{cases}\frac{z}{\varepsilon}, & z \leqslant \varepsilon \\ 1, & \text { иначе. }\end{cases}
$$

УтВеРЖДЕНИЕ 2.1. Если поле $\mathbb{T}$ обладает свойствами В1-В3, функиии $\varphi_{\varepsilon}-$ свойствами D1-D3, а тензор $\mathbb{P}$ - свойствами A1-A4, то формула (2.1) дает тензори $\mathbb{P}_{b \varepsilon}$, удовлетворяющие $\mathrm{C} 1-\mathrm{C} 5$. 
ДоказАтельСтво. Свойства $\mathrm{C} 1, \mathrm{C} 2$ и $\mathrm{C} 4$ очевидны, поскольку $\varkappa(\mathbb{D}) \geqslant \mu_{0}|\mathbb{D}|$. Далее, при $|\mathbb{D}(\mathbf{v})| \in\left[\delta / \mu_{0}, d_{0}\right]$ получаем $\varkappa(\mathbb{D}(\mathbf{v})) \in\left[\delta, d_{0}\right]$, что в силу D3 и оценки $\left|\mathbb{P}_{b}\right| \leqslant p_{*}$ дает С3. Остается проверить аксиомы A1-A3 для $\mathbb{P}+\mathbb{P}_{b \varepsilon}$ и $\mathrm{A} 2-\mathrm{A} 4$ для $\mathbb{P}_{b \varepsilon}$. Заметим, что

$$
\mathbb{P}_{b \varepsilon}: \mathbb{D}=p_{*} \varphi_{\varepsilon}(\varkappa(\mathbb{D})) \varkappa(\mathbb{D}) \geqslant 0,
$$

откуда следует A1. Далее, выбрав произвольно поля $\mathbf{u}_{1,2} \in X$ и обозначив $\mathbb{D}_{i}=$ $\mathbb{D}\left(\mathbf{u}_{i}\right)$, будем иметь (ввиду В3) в случае $\mathbb{D}_{1,2} \neq 0$

$$
\begin{aligned}
\mathbb{P}_{b \varepsilon}\left(\mathbf{u}_{i}\right): \mathbb{D}_{j} & =\varphi_{\varepsilon}\left(\varkappa\left(\mathbb{D}_{i}\right)\right) p_{*} \mathbb{T}\left(\frac{\mathbb{D}_{i}}{\left|\mathbb{D}_{i}\right|}\right): \frac{\mathbb{D}_{j}}{\left|\mathbb{D}_{j}\right|}\left|\mathbb{D}_{j}\right| \\
& \leqslant \varphi_{\varepsilon}\left(\varkappa\left(\mathbb{D}_{i}\right)\right) p_{*} \mathbb{T}\left(\frac{\mathbb{D}_{j}}{\left|\mathbb{D}_{j}\right|}\right): \frac{\mathbb{D}_{j}}{\left|\mathbb{D}_{j}\right|}\left|\mathbb{D}_{j}\right| \\
& =p_{*} \varphi_{\varepsilon}\left(\varkappa\left(\mathbb{D}_{i}\right)\right) \mu\left(\frac{\mathbb{D}_{j}}{\left|\mathbb{D}_{j}\right|}\right)\left|\mathbb{D}_{j}\right|=p_{*} \varphi_{\varepsilon}\left(\varkappa\left(\mathbb{D}_{i}\right)\right) \varkappa\left(\mathbb{D}_{j}\right),
\end{aligned}
$$

причем в силу D1 и $\mathrm{C} 2$ полученное неравенство сохраняется (в виде равенства) и в случае $\mathbb{D}_{1}=0$ или $\mathbb{D}_{2}=0$. Но теперь ввиду $\mathrm{D} 2$ и $(2.2)$ монотонность $\mathbb{P}_{b \varepsilon}$, а значит, и $\mathbb{P}+\mathbb{P}_{b \varepsilon}$, очевидна.

В силу тривиальной оценки $\left|\mathbb{P}+\mathbb{P}_{b \varepsilon}\right| \leqslant 2\left(\left(|\mathbb{P}|+p_{*}\right) / 2\right)$ и $\Delta_{2}$-условия для $\bar{M}$ мы можем написать

$$
\bar{M}\left(\left|\mathbb{P}+\mathbb{P}_{b \varepsilon}\right|\right) \leqslant C_{1} \frac{\bar{M}(|\mathbb{P}|)+\bar{M}\left(p_{*}\right)}{2},
$$

что после интегрирования по $\Omega$ даст А3.

Наконец, А4 для $\mathbb{P}_{b \varepsilon}$ следует из теоремы Лебега: $\mathbb{D}\left(\mathbf{u}-\varepsilon_{1} \mathbf{v}\right) \rightarrow \mathbb{D}(\mathbf{u})$ п.в. при $\varepsilon_{1} \rightarrow 0$, откуда $\mathbb{P}_{b \varepsilon}\left(\mathbf{u}-\varepsilon_{1} \mathbf{v}\right) \rightarrow \mathbb{P}_{b \varepsilon}(\mathbf{u})$ п.в., и имеется оценка $\left|\mathbb{P}_{b \varepsilon}\right| \leqslant p_{*}$, так что сходимость сильная в любом $L_{p}$.

Теперь рассмотрим вопрос о нетривиальных парах $(\mathscr{P}, \mathbb{T})$, удовлетворяющих аксиомам B1-B3, что эквивалентно построению векторного поля $\mathbf{t}$ на единичной сфере в $\mathbb{R}^{m}$ (где $\left.m=n(n+1) / 2\right)$, обеспечивающего В1-В3 и такого, что множество его значений д $\mathscr{P}$ определяет область $\mathscr{P} \subset \mathbb{R}^{m}$ со свойствами, оговоренными во введении. Удобно сперва проанализировать эту задачу на модельном случае $m=2$ (хотя он и не соответствует никаким $\left.\mathbb{S}_{n}\right)$, а затем уже́ обобщить на произвольные $m>2$.

Итак, ищем поле $\mathbf{t}$, заданное на единичной окружности $\{(\cos \varphi, \sin \varphi) \mid \varphi \in[0,2 \pi]\}$, в виде

$$
\mathbf{t}(\varphi)=r(\varphi)\left[\begin{array}{l}
\cos (\beta(\varphi)+\varphi) \\
\sin (\beta(\varphi)+\varphi)
\end{array}\right] .
$$

Для того, чтобы с ростом $\varphi$ вектор $\mathbf{t}(\varphi)$ пробегал границу выпуклой области $\mathscr{P} \in \mathbb{R}^{2}$, необходимо условие

Е1) функция $(\beta(\varphi)+\varphi)$ возрастает по $\varphi$.

Определив монотонную функцию $\alpha$ формулой $\alpha^{-1}(\varphi)=\beta(\varphi)+\varphi$, получаем, что

$$
\mathbf{t}(\alpha(\varphi))=r(\alpha(\varphi))\left[\begin{array}{l}
\cos \varphi \\
\sin \varphi
\end{array}\right],
$$

T.e.

$$
\partial \mathscr{P}=\left\{r=r_{1}(\varphi) \equiv r(\alpha(\varphi)) \mid \varphi \in[0,2 \pi]\right\} .
$$


Аксиома В1 и свойства $0 \in \mathscr{P}, \rho(0, \partial \mathscr{P})=1$ приводят к требованиям

E2) $r \in C ; r(2 \pi)=r(0) ; \beta \in C ; 0<r \leqslant 1 ; \max r=1$.

Кроме того, В1 дает $\beta(2 \pi)-\beta(0)=2 \pi k, k \in \mathbb{Z}$, но поскольку

$$
\mu(\varphi)=r(\varphi) \cos \beta(\varphi),
$$

то (ввиду непрерывности $\beta$ ) В2 дает $\beta \in(-\pi / 2+2 \pi q, \pi / 2+2 \pi q), q \in \mathbb{Z}$, так что обязательно $k=0$. Итак (полагая без ограничения общности $q=0$ ),

Е3) $\beta(2 \pi)=\beta(0), \beta \in(-\pi / 2, \pi / 2)$.

Если считать $\beta$ и $r$ достаточно гладкими, то условие выпуклости $\mathscr{P}$ записывается в виде $r_{1} r_{1}^{\prime \prime} \leqslant r_{1}^{2}+2 r_{1}^{\prime 2}$, что после элементарного пересчета принимает форму неравенства

$$
r r^{\prime \prime} \leqslant r^{2}\left(\beta^{\prime}+1\right)^{2}+2 r^{2}+r r^{\prime} \frac{\beta^{\prime \prime}}{\beta^{\prime}+1} .
$$

Свойство В3 принимает вид $r\left(\varphi_{2}\right) \cos \left(\beta\left(\varphi_{2}\right)+\varphi_{2}-\varphi_{1}\right) \leqslant r\left(\varphi_{1}\right) \cos \beta\left(\varphi_{1}\right)$, или, после замены $\varphi_{2}=\varphi, \varphi_{1}=\varphi+\lambda$, без ограничения общности считая, что $\left(\varphi_{1}-\varphi_{2}\right) \in[-\pi, \pi]$, получаем окончательную форму

E4) $r(\varphi) \cos (\beta(\varphi)-\lambda) \leqslant r(\varphi+\lambda) \cos \beta(\varphi+\lambda), \forall \varphi \in \mathbb{R}, \lambda \in[-\pi, \pi]$.

Рассматривая свойство Е4 при малых $\lambda$, легко получить необходимое условие

$$
\frac{r^{\prime}}{r}=(\operatorname{tg} \beta)\left(\beta^{\prime}+1\right),
$$

из которого легко следует (2.5) (причем строго), т.е. ЯР всегда строго выпукла в описанных условиях.

Таким образом, наша задача (т.е. E1-E4) переформулируется следующим эквивалентным образом. Мы произвольно задаем функцию $\gamma$ со свойствами

F1) $\gamma$ непрерывна на $\mathbb{R}$ и периодична с периодом $2 \pi$;

F2) $\gamma^{\prime}+\gamma^{2}+1 \geqslant 0$

F3) $\int_{0}^{2 \pi} \gamma(\varphi) d \varphi=0$;

и вычисляем

$$
\beta=\operatorname{arctg} \gamma, \quad r(\varphi)=C \sqrt{\gamma^{2}(\varphi)+1} \cdot \exp \left(\int_{0}^{\varphi} \gamma(\eta) d \eta\right)
$$

(т.е. решаем (2.6)), что при подходящем выборе $C>0$ гарантирует выполнение условий Е1-Е3 и (2.5), так что остается лишь обеспечить Е4. При этом

$$
\mu(\varphi)=C \exp \left(\int_{0}^{\varphi} \gamma(\eta) d \eta\right)
$$

а условие Е4 принимает вид

F4) $\exp \left(\int_{\varphi}^{\varphi+\lambda} \gamma(\eta) d \eta\right) \geqslant \cos \lambda+\gamma(\varphi) \sin \lambda$ для всех $\varphi \in \mathbb{R}, \lambda \in[-\pi, \pi]$.

Множество функций $\gamma$ со свойствами F1-F4 включает "почти все" функции со свойствами F1 и F3, как показывает следующее

УтВеРЖДЕНИЕ 2.2. Пусть функиия $\gamma$ удовлетворяет F1, F3 и, кроме того, $\left.\mathrm{F} 2^{\prime}\right) \gamma^{\prime} \geqslant-1$

Тогда $\gamma$ удовлетворяет также $\mathrm{F} 2$ и $\mathrm{F} 4$. 
ЛЕмма 2.3. Функиия

$$
F(\lambda, \gamma)=\exp \left(\lambda \gamma-\frac{\lambda^{2}}{2}\right)-\cos \lambda-\gamma \sin \lambda
$$

неотрицательна при всех $(\lambda, \gamma) \in[-\pi, \pi] \times \mathbb{R}$.

ДоКАЗАТЕЛЬСТво ЛЕммы 2.3 не приводим ввиду его элементарности.

ДоКАЗАТЕЛЬСТво УТВЕРЖДЕНИЯ 2.2. Из F2' очевидно следует F2, а также оценки

$$
\begin{array}{lll}
\gamma(\eta)-\gamma(\varphi) \geqslant-(\eta-\varphi) & \text { при } \quad \eta \geqslant \varphi, \\
\gamma(\eta)-\gamma(\varphi) \leqslant-(\eta-\varphi) & \text { при } \quad \eta \leqslant \varphi .
\end{array}
$$

Отсюда, очевидно,

$$
\int_{\varphi}^{\varphi+\lambda}(\gamma(\eta)-\gamma(\varphi)) d \eta \geqslant-\int_{\varphi}^{\varphi+\lambda}(\eta-\varphi) d \eta=-\frac{\lambda^{2}}{2},
$$

т.e.

$$
\exp \left(\int_{\varphi}^{\varphi+\lambda} \gamma(\eta) d \eta\right)-\cos \lambda-\gamma(\varphi) \sin \lambda \geqslant F(\lambda, \gamma(\varphi))
$$

что завершает доказательство ввиду леммы 2.3 .

Итак, модельная задача о плоских полях $\mathbf{t}$ допускает богатое семейство решений (и достаточно точное описание всех возможных решений), на основе которых мы можем предъявить класс осесимметрических (т.е. специальных, не претендуя на полное решение задачи о всех возможных $(\mathscr{P}, \mathbb{T}))$ пар $(\mathscr{P}, \mathbb{T})$ в любом $\mathbb{R}^{m}$ (в том числе в $\left.\mathbb{S}_{n}\right)$. Для этого зафиксируем произвольно элемент $\mathbb{B}_{0} \in S_{1}$ (полюс) и соответствующий ему экватор $\left\{\mathbb{C} \in S_{1} \mid \mathbb{C}: \mathbb{B}_{0}=0\right\}$. Любой элемент $\mathbb{B}_{1} \in S_{1}$, не совпадающий с $\pm \mathbb{B}_{0}$, имеет единственный соответствующий ему экваториальный элемент

$$
\mathbb{E}\left(\mathbb{B}_{1}\right)=\frac{\mathbb{B}_{1}-\left(\mathbb{B}_{1}: \mathbb{B}_{0}\right) \mathbb{B}_{0}}{\left|\mathbb{B}_{1}-\left(\mathbb{B}_{1}: \mathbb{B}_{0}\right) \mathbb{B}_{0}\right|}
$$

и определяет свой меридиан как совокупность всех $\mathbb{B} \in S_{1}$ с тем же $\mathbb{E}(\mathbb{B})$. Введя широту любого тензора $\mathbb{B} \in S_{1}$ как угол $\varphi(\mathbb{B})=\arccos \left(\mathbb{B}: \mathbb{B}_{0}\right)$ из интервала $[0, \pi]$ (широта экватора тогда равна $\pi / 2$, полюса $\mathbb{B}_{0}-$ нулю, а полюса $\left(-\mathbb{B}_{0}\right)$ равна $\pi$ ), мы легко убеждаемся в геометрически очевидных равенствах

$$
\sin \varphi=\mathbb{E}(\mathbb{B}): \mathbb{B}, \quad \mathbb{B}=(\cos \varphi) \mathbb{B}_{0}+(\sin \varphi) \mathbb{E}(\mathbb{B}),
$$

откуда $\sin \varphi=\left|\mathbb{B}-(\cos \varphi) \mathbb{B}_{0}\right|$. Теперь мы готовы предъявить анонсированный класс пар $(\mathscr{P}, \mathbb{T})$.

УтВЕРЖДЕНИЕ 2.4. Пусть функиии г и $\beta$ обладают свойствами Е1-Е4 и, кроме moго,

E5) $\beta(2 \pi-\varphi)=-\beta(\varphi), r(2 \pi-\varphi)=r(\varphi), \varphi \in[0, \pi]$.

Тогда осесимметрическое поле

$$
\mathbb{T}(\mathbb{B})=r(\varphi) \cos (\beta(\varphi)+\varphi) \mathbb{B}_{0}+r(\varphi) \sin (\beta(\varphi)+\varphi) \mathbb{E}(\mathbb{B})
$$

обладает свойствами В1-В3, а определяемое им Я્Р есть ограниченная выпуклая область в $\mathbb{S}_{n}$, содержащая 0, причем $\rho(0, \partial \mathscr{P})=1$. 
ДокАЗАтЕЛьСтво. Из Е3 и Е5 следует $\beta(0)=\beta(\pi)=0$, откуда вытекает непрерывность $\mathbb{T}$ на полюсах, в то время как в остальных точках $S_{1}$ непрерывность $\mathbb{T}$ очевидна из Е2. Свойства $\mathscr{P}$ получаются из соответствующих (обеспеченных в силу E1-E4) свойств сечений $\mathscr{P}$ меридианами (т.е. имеет место (2.3)). Нетрудно проверить, что (2.4) снова верно, что обеспечивает В2 в силу Е2 и Е3. Ввиду Е1 имеем $0 \leqslant \beta(\varphi)+\varphi \leqslant \pi$ при $\varphi \in[0, \pi]$, поэтому на основании Е4 можно написать

$$
\begin{aligned}
\mathbb{T}\left(\mathbb{B}_{2}\right): \mathbb{B}_{1}= & r\left(\varphi_{2}\right) \cos \left(\beta\left(\varphi_{2}\right)+\varphi_{2}\right) \cos \varphi_{1} \\
& \quad+r\left(\varphi_{2}\right) \cos \left(\beta\left(\varphi_{2}\right)+\varphi_{2}\right)\left(\sin \varphi_{1}\right) \mathbb{E}\left(\mathbb{B}_{2}\right): \mathbb{E}\left(\mathbb{B}_{1}\right) \\
\leqslant & r\left(\varphi_{2}\right) \cos \left(\beta\left(\varphi_{2}\right)+\varphi_{2}-\varphi_{1}\right) \leqslant r\left(\varphi_{1}\right) \cos \beta\left(\varphi_{1}\right)=\mathbb{T}\left(\mathbb{B}_{1}\right): \mathbb{B}_{1} .
\end{aligned}
$$

Чтобы описать множество функций $(\beta, r)$ со свойствами Е1-Е5, воспользуемся снова представлением (2.7).

УтВЕРЖДЕНИЕ 2.5. Пусть функиия $\gamma$ удовлетворяет F1, F2' u

F5) $\gamma(2 \pi-\varphi)=-\gamma(\varphi), \varphi \in[0, \pi]$.

Тогда $\beta$ и г, вычисленные из (2.7) с подходящей $C>0$, удовлетворяют E1-Е5.

ДокАЗАтЕЛьСтво. Из F5 следует F3, так что можно применить утверждение 2.2 , после чего построить $\beta$ и $r$ из (2.7), удовлетворяющие E1-E4. Теперь Е5 легко проверяется непосредственно в (2.7) на основании F5 и F3.

ЗАмечание 2.6. Из F5 и F1 следует $\gamma(0)=\gamma(\pi)=\gamma(2 \pi)=0$, причем на самом деле $\mathrm{F} 2^{\prime}$ означает $\left|\gamma^{\prime}\right| \leqslant 1$. В утверждении $(2.5)$ описан частный случай. Вообще говоря, следует требовать вместо $\mathrm{F} 2^{\prime}$ оценку $\left|\gamma^{\prime}\right| \leqslant 1+\gamma^{2}$ (в которую превращается F2 ввиду F5) и отдельно проверять F4.

ЗАМЕЧАНИЕ 2.7. Упомянутый во введении изотропный случай соответствует $\beta \equiv$ $\gamma \equiv 0, r \equiv 1$.

3. Разрешимость задачи А. Прежде чем формулировать основную теорему, определим необходимые вспомогательные понятия.

ОПРЕДЕЛЕНИЕ 3.1. Задачей $\mathrm{A}_{\varepsilon}$ назовем задачу (0.1), (0.2), (1.1), (1.2), замыкаемую соотношением $\mathbb{P}_{r}=\mathbb{P}_{f}+\mathbb{P}_{b \varepsilon}$, где $\mathbb{P}_{b \varepsilon}$ - аппроксимирующее семейство стоксовых тензоров со свойствами C1-C5 (а значит, и С6-C8).

Как показано в п. 2 , требуемое в определении 3.1 семейство $\mathbb{P}_{b \varepsilon}$ всегда может быть построено - например, по формуле (2.1) с $\varphi_{\varepsilon}$ из D1-D3.

Определим пространство

$$
Y=\left\{\mathbf{v}\left|\mathbb{D}(\mathbf{v}) \in L_{M}\left(Q_{T}\right), \mathbf{v}\right|_{\partial \Omega \times(0, T)}=0\right\}
$$

с нормой $\|\mathbf{v}\|_{Y}=\|\mathbb{D}(\mathbf{v})\|_{L_{M}\left(Q_{T}\right)}$ и $\mathrm{N}$-функции

$$
\Phi_{\gamma}(s)=(1+s) \ln ^{\gamma}(1+s), \quad \Psi_{\gamma}=\bar{\Phi}_{\gamma} .
$$

Свойства пространства $Y$ изучались в [9], [10], где, в частности, показано, что оно не обязано совпадать с $L_{M}(0, T, X)$. Будем налагать следующие ограничения на входные данные задачи А:

$$
\mathbf{f} \in K_{\Psi_{\beta / 2}}\left(Q_{T}\right), \quad \rho_{0} \in L_{\Phi_{\beta}}(\Omega), \quad \frac{\mathbf{w}_{0}}{\rho_{0}} \in L_{\Psi_{\beta / 2}}\left(\operatorname{supp} \rho_{0}\right), \quad \beta>\frac{7}{2} .
$$


В этих условиях благодаря С5 задача А $\varepsilon$ удовлетворяет условиям теоремы 3.5 из [10], что дает существование ее решения $\left(\rho_{\varepsilon}, \mathbf{u}_{\varepsilon}\right)$ при любом $T>0$ в классе

$$
\rho_{\varepsilon} \in L_{\infty}\left(0, T, L_{\Phi_{\beta}}(\Omega)\right), \quad \rho_{\varepsilon} \geqslant 0, \quad \mathbf{u}_{\varepsilon} \in Y,
$$

а также энергетическое равенство

$$
\left.\int_{\Omega}\left(\frac{\rho_{\varepsilon}\left|\mathbf{u}_{\varepsilon}\right|^{2}}{2}+\rho_{\varepsilon} \ln \rho_{\varepsilon}\right) d \mathbf{x}\right|_{0} ^{t}+\int_{0}^{t} \int_{\Omega}\left[\left(\mathbb{P}\left(\mathbf{u}_{\varepsilon}\right)+\mathbb{P}_{b \varepsilon}\left(\mathbf{u}_{\varepsilon}\right)\right): \mathbb{D}\left(\mathbf{u}_{\varepsilon}\right)-\rho_{\varepsilon} \mathbf{u}_{\varepsilon} \cdot \mathbf{f}\right] d \mathbf{x} d s=0 .
$$

Теперь мы готовы сформулировать основной результат.

Теорема 3.2. Пусть заданы тензорная функиия $\mathbb{P}(\cdot)$ со свойствами А1-А7 с ограничением (1.3) (например, в виде, описанном в примере 1.1) и поле $\mathbb{T}$ со свойствами В1-В3 (например, в виде, описанном в п.2, сюда входит и тривиальныи изотропный случай тождественного поля $\mathbb{T}$ ). Пусть произвольно задань число $T>0$ и входные данные класса (3.1). Тогда выполнены следующие утверждения.

1. Задача А (см. определение 1.3) имеет в $Q_{T}$ решение класса

$$
\rho \in L_{\infty}\left(0, T, L_{\Phi_{\beta}}(\Omega)\right), \quad \rho \geqslant 0, \quad \mathbf{u} \in Y
$$

удовлетворяющее энергетическому равенству

$$
\left.\int_{\Omega}\left(\frac{\rho|\mathbf{u}|^{2}}{2}+\rho \ln \rho\right) d \mathbf{x}\right|_{0} ^{t}+\int_{0}^{t} \int_{\Omega}\left[\left(\mathbb{P}(\mathbf{u})+\mathbb{P}_{b}(\mathbf{u})\right): \mathbb{D}(\mathbf{u})-\rho \mathbf{u} \cdot \mathbf{f}\right] d \mathbf{x} d s=0 .
$$

2. Уравнение (0.2) (с (0.3), (0.4) и (1.2)) удовлетворяется этим решением в том смысле, ито $\mathbb{P}_{b}(\mathbf{u})=p_{*} \mathbb{T}(\mathbb{D} /|\mathbb{D}|)$ при $\mathbb{D} \neq 0$, а на множестве $\{\mathbb{D}=0\}$ величина $\mathbb{P}_{b}(\mathbf{u})$ есть вполне определенный тензор $\overline{\mathbb{P}}_{b}$ со значениями из множества $p_{*} \overline{\mathscr{P}}$.

3. Решение $(\rho, \mathbf{u})$ есть предел решений $\left(\rho_{\varepsilon_{k}}, \mathbf{u}_{\varepsilon_{k}}\right)$ некоторой последовательности задач $A_{\varepsilon_{k}}$, сходящихся к нему в смысле $(3.9)$ и сильно в $L_{p}\left(0, T, L_{\Phi_{*}}(\Omega)\right) \times L_{p}\left(Q_{T}\right)$ с любыми $p<+\infty, \Phi_{*} \prec \prec \Phi_{\beta}$, причем $\rho_{\varepsilon_{k}} \rightarrow \rho, \mathbb{D}\left(\mathbf{u}_{\varepsilon_{k}}\right) \rightarrow \mathbb{D}(\mathbf{u})$ п.в. в $Q_{T}$.

4. Уравнение (0.1) выполнено для рассматриваемого решения в пространстве $L_{M}\left(0, T, W^{-1} L_{\Phi_{\beta}}(\Omega)\right)$, a $(0.2)$ - в $Y^{*}$, начальные данные $(1.1)_{1,2}$ принимаются по сильной непрерывности в пространстве $W^{-1} L_{\Phi_{\beta}}(\Omega) \times X^{*}$, а краевое условие $(1.1)_{3}$ понимается в смысле значений непрерывной по Гёльдеру (по х при всех $t$ ) функции.

ЗАмечАниЕ 3.3. Пункт 4 теоремы 3.2 не нуждается в отдельном доказательстве, так как требуемые свойства решения показываются аналогично тому, как это делалось в [10].

ДоКАЗАТЕЛЬСтво теоремы 3.2. Как сказано выше, условия (3.1) обеспечивают существование решения $\left(\rho_{\varepsilon}, \mathbf{u}_{\varepsilon}\right)$ задачи $\mathrm{A}_{\varepsilon}$ со свойствами (3.2), (3.3). Обоснование предельного перехода по $\varepsilon \rightarrow 0$ для удобства представим в виде нескольких этапов.

Этап I: равномерные по в оценки, слабые пределы. Применяя к задаче $(0.1),(1.1)_{1}$ для $\left(\rho_{\varepsilon}, \mathbf{u}_{\varepsilon}\right)$ результаты [13], получим оценку

$$
\left\|\rho_{\varepsilon}\right\|_{L_{\infty}\left(0, t, L_{\Phi_{\beta}}(\Omega)\right)} \leqslant C_{1}\left(1+\int_{0}^{t} \int_{\Omega} M\left(\operatorname{div} \mathbf{u}_{\varepsilon}\right) d \mathbf{x} d s\right)
$$

(здесь и далее символами $C_{k}, k \in \mathbb{N}$, обозначены постоянные, зависящие лишь от $T$, mes $\Omega$ и норм (3.1)). При этом следует оговориться, что в целом работа [13] посвящена периодической по х задаче Коши (с параллелепипедом $\Omega$ вместо произвольной $\Omega$ 
и условий $\left.(1.1)_{3}\right)$, но, как нетрудно проследить, нужный нам результат (3.5) сохраняется и в нашей ситуации. Используя теперь очевидные неравенства

$$
-e^{-1} \leqslant \xi \ln \xi \leqslant \Phi_{\beta}(\xi)+1 \quad \forall \xi \geqslant 0, \quad \rho_{\varepsilon} \mathbf{u}_{\varepsilon} \cdot \mathbf{f} \leqslant \varepsilon_{1} \frac{\rho_{\varepsilon}|\mathbf{f}|^{2}}{2}+\frac{\rho_{\varepsilon}\left|\mathbf{u}_{\varepsilon}\right|^{2}}{2 \varepsilon_{1}} \quad \forall \varepsilon_{1}>0,
$$

аксиому $\mathrm{A} 1$ для $\mathbb{P}+\mathbb{P}_{b \varepsilon}$ и подбирая $\varepsilon_{1}$ достаточно малым в соответствии с $C_{1}$ и нормой $\mathbf{f}$, легко выводим из (3.3) оценку

$$
\int_{0}^{T} \int_{\Omega} M\left(\left|\mathbb{D}\left(\mathbf{u}_{\varepsilon}\right)\right|\right) d \mathbf{x} d t \leqslant C_{2}
$$

а тогда (3.5) дает

$$
\left\|\rho_{\varepsilon}\right\|_{L_{\infty}\left(0, T, L_{\Phi_{\beta}}(\Omega)\right)} \leqslant C_{3} .
$$

Применяя теперь свойства $\mathrm{A} 3$ для $\mathbb{P}$ и $\mathrm{C} 1$ для $\mathbb{P}_{b \varepsilon}$, мы можем написать

$$
\int_{0}^{T} \int_{\Omega} \bar{M}\left(\left|\mathbb{P}\left(\mathbf{u}_{\varepsilon}\right)\right|\right) d \mathbf{x} d t \leqslant C_{4}, \quad\left|\mathbb{P}_{b \varepsilon}\left(\mathbf{u}_{\varepsilon}\right)\right| \leqslant C_{5}
$$

Оценки (3.6)-(3.8) позволяют выделить последовательности (сохраним для них тот же индекс $\varepsilon$ ) такие, что

$$
\begin{aligned}
& \left(\rho_{\varepsilon}, \mathbf{u}_{\varepsilon}, \mathbb{P}\left(\mathbf{u}_{\varepsilon}\right), \mathbb{P}_{b \varepsilon}\left(\mathbf{u}_{\varepsilon}\right)\right) \rightarrow\left(\rho, \mathbf{u}, \overline{\mathbb{P}}, \overline{\mathbb{P}}_{b}\right) \\
& \quad \text { *-слабо в } L_{\infty}\left(0, T, L_{\Phi_{\beta}}(\Omega)\right) \times Y \times L_{\bar{M}}\left(Q_{T}\right) \times L_{\infty}\left(Q_{T}\right),
\end{aligned}
$$

где

$$
\rho \in L_{\infty}\left(0, T, L_{\Phi_{\beta}}(\Omega)\right), \quad \mathbf{u} \in Y, \quad \overline{\mathbb{P}} \in L_{\bar{M}}\left(Q_{T}\right), \quad \overline{\mathbb{P}}_{b} \in L_{\infty}\left(Q_{T}\right),
$$

- некоторые функции. Кроме того, аналогично [10] строятся *-слабые пределы $\rho_{\varepsilon} \mathbf{u}_{\varepsilon} \rightarrow \mathbf{a}, \rho_{\varepsilon} \mathbf{u}_{\varepsilon} \otimes \mathbf{u}_{\varepsilon} \rightarrow \mathbb{B}$ и доказывается, что $\mathbf{a}=\rho \mathbf{u}, \mathbb{B}=\rho \mathbf{u} \otimes \mathbf{u}$, причем

$$
\begin{gathered}
\rho_{\varepsilon} \rightarrow \rho, \quad \rho_{\varepsilon} \mathbf{u}_{\varepsilon} \rightarrow \rho \mathbf{u}, \quad \rho_{\varepsilon} \mathbf{u}_{\varepsilon} \otimes \mathbf{u}_{\varepsilon} \rightarrow \rho \mathbf{u} \otimes \mathbf{u} \\
\text { *-слабо в } L_{\Phi_{\beta}}(\Omega) \text { при п.в. } t \in[0, T] .
\end{gathered}
$$

Благодаря (3.9) и (3.11) в задаче $\mathrm{A}_{\varepsilon}$ можно перейти к пределу и получить уравнение (0.1) и вместо (0.2) уравнение

$$
\frac{\partial(\rho \mathbf{u})}{\partial t}+\operatorname{div}(\rho \mathbf{u} \otimes \mathbf{u})=-\nabla \rho+\operatorname{div} \overline{\mathbb{P}}+\operatorname{div} \overline{\mathbb{P}}_{b}+\rho \mathbf{f},
$$

понимаемые в соответствующем обобщенном смысле. Таким образом, для того, чтобы доказать, что пара $(\rho, \mathbf{u})$ есть решение задачи $\mathrm{A}$, остается обосновать равенства

$$
\operatorname{div} \overline{\mathbb{P}}=\operatorname{div} \mathbb{P}(\mathbf{u}), \quad \operatorname{div} \overline{\mathbb{P}}_{b}=\operatorname{div} \mathbb{P}_{b}(\mathbf{u}) .
$$

Этап II: сравнение энергетических равенств. Переходя в (3.3) к пределу на основании (3.9) и (3.11), мы можем написать

$$
\begin{gathered}
\left.\int_{\Omega} \frac{\rho|\mathbf{u}|^{2}}{2} d \mathbf{x}\right|_{0} ^{t}+\lim _{\varepsilon \rightarrow 0}\left(\int_{0}^{t} \int_{\Omega}\left(\mathbb{P}\left(\mathbf{u}_{\varepsilon}\right)+\mathbb{P}_{b \varepsilon}\left(\mathbf{u}_{\varepsilon}\right)\right): \mathbb{D}\left(\mathbf{u}_{\varepsilon}\right) d \mathbf{x} d s+K\left(\rho_{\varepsilon}\right)\right) \\
=\int_{0}^{t} \int_{\Omega} \rho \mathbf{u} \cdot \mathbf{f} d \mathbf{x} d s+K\left(\rho_{0}\right)
\end{gathered}
$$


где $K(\rho)=\int_{\Omega} \rho \ln \rho d \mathbf{x}$. С другой стороны, по лемме 3.3 из [10] решение задачи $(0.1),(3.12),(1.1)$ класса (3.10) обязано удовлетворять равенству

$$
\left.\int_{\Omega} \frac{\rho|\mathbf{u}|^{2}}{2} d \mathbf{x}\right|_{0} ^{t}+\int_{0}^{t} \int_{\Omega}\left(\overline{\mathbb{P}}+\overline{\mathbb{P}}_{b}\right): \mathbb{D}(\mathbf{u}) d \mathbf{x} d s+K(\rho)=\int_{0}^{t} \int_{\Omega} \rho \mathbf{u} \cdot \mathbf{f} d \mathbf{x} d s+K\left(\rho_{0}\right) .
$$

Используя вытекающее из свойства С8 соотношение

$$
\lim _{\varepsilon \rightarrow 0} \int_{0}^{t} \int_{\Omega}\left(\mathbb{P}_{b \varepsilon}\left(\mathbf{u}_{\varepsilon}\right)-\mathbb{P}_{b 0}\left(\mathbf{u}_{\varepsilon}\right)\right): \mathbb{D}\left(\mathbf{u}_{\varepsilon}\right) d \mathbf{x} d s=0,
$$

мы можем заключить из (3.13) и (3.14), что

$$
\lim _{\varepsilon \rightarrow 0}\left(N\left(\mathbf{u}_{\varepsilon}\right)+K\left(\rho_{\varepsilon}\right)\right)=\int_{0}^{t} \int_{\Omega}\left(\overline{\mathbb{P}}+\overline{\mathbb{P}}_{b}\right): \mathbb{D}(\mathbf{u}) d \mathbf{x} d s+K(\rho) .
$$

Как указано в примере 4.4 , функционал $K$ *-слабо полунепрерывен снизу, и ввиду $(3.11)_{1}$ мы можем утверждать, что $K(\rho) \leqslant \underline{\lim } K\left(\rho_{\varepsilon}\right)$, так что $(3.16)$, в частности, дает

$$
\varlimsup_{\varepsilon \rightarrow 0} N\left(\mathbf{u}_{\varepsilon}\right) \leqslant \int_{0}^{t} \int_{\Omega}\left(\overline{\mathbb{P}}+\overline{\mathbb{P}}_{b}\right): \mathbb{D}(\mathbf{u}) d \mathbf{x} d s .
$$

Этап III: соображения монотонности. В силу аксиомы А2 для $\mathbb{P}+\mathbb{P}_{b \varepsilon}$ при всех $\mathbf{v} \in Y$ можно написать неравенство

$$
\begin{aligned}
& \int_{0}^{t} \int_{\Omega}\left(\mathbb{P}+\mathbb{P}_{b \varepsilon}\right)\left(\mathbf{u}_{\varepsilon}\right): \mathbb{D}\left(\mathbf{u}_{\varepsilon}\right) d \mathbf{x} d s \\
& \quad \geqslant \int_{0}^{t} \int_{\Omega}\left(\mathbb{P}+\mathbb{P}_{b \varepsilon}\right)(\mathbf{v}):\left(\mathbb{D}\left(\mathbf{u}_{\varepsilon}\right)-\mathbb{D}(\mathbf{v})\right) d \mathbf{x} d s+\int_{0}^{t} \int_{\Omega}\left(\mathbb{P}+\mathbb{P}_{b \varepsilon}\right)\left(\mathbf{u}_{\varepsilon}\right): \mathbb{D}(\mathbf{v}) d \mathbf{x} d s
\end{aligned}
$$

в котором можно перейти к пределу:

$$
\underline{\lim } N\left(\mathbf{u}_{\varepsilon}\right) \geqslant \int_{0}^{t} \int_{\Omega}\left(\mathbb{P}+\mathbb{P}_{b 0}\right)(\mathbf{v}):(\mathbb{D}(\mathbf{u})-\mathbb{D}(\mathbf{v})) d \mathbf{x} d s+\int_{0}^{t} \int_{\Omega}\left(\overline{\mathbb{P}}+\overline{\mathbb{P}}_{b}\right): \mathbb{D}(\mathbf{v}) d \mathbf{x} d s .
$$

Здесь мы воспользовались соотношениями (3.9) и (3.15), а также сильной сходимостью (в любом $\left.L_{p}\left(Q_{T}\right)\right) \mathbb{P}_{b \varepsilon}(\mathbf{v}) \rightarrow \mathbb{P}_{b 0}(\mathbf{v})$, следующей из С1 и С6. Теперь (3.17) и (3.18) позволяют записать неравенство

$$
\int_{0}^{t} \int_{\Omega}\left[\left(\overline{\mathbb{P}}+\overline{\mathbb{P}}_{b}\right)-\left(\mathbb{P}+\mathbb{P}_{b 0}\right)(\mathbf{v})\right]:(\mathbb{D}(\mathbf{u})-\mathbb{D}(\mathbf{v})) d \mathbf{x} d s \geqslant 0,
$$

которое, однако, не допускает применения стандартных аргументов метода монотонности $\left(\mathbf{v}=\mathbf{u}-\varepsilon_{1} \mathbf{w}\right.$, и т.д. [14; с. 172$\left.]\right)$ в силу разрывности тензора $\mathbb{P}_{b 0}$ (т.е. поточечный $\lim _{\varepsilon_{1} \rightarrow 0} \mathbb{P}_{b 0}\left(\mathbf{u}+\varepsilon_{1} \mathbf{w}\right)$ зависит от $\mathbf{w}$ и, вообе говоря, не равен $\left.\mathbb{P}_{b 0}(\mathbf{u})\right)$, так что следует действовать несколько иначе, используя непрерывность тензора $\mathbb{P}_{b 0}$ на лучах

$$
\mathbb{P}_{b 0}\left(\mathbf{v} \pm \varepsilon_{1} \mathbf{v}\right) \rightarrow \mathbb{P}_{b 0}(\mathbf{v}) \quad \text { при всех } \mathbf{v} \text { (поточечно) }
$$

Этап IV: частичное использование (3.19) и "снятие черт" в (3.14) и (3.16); сильная сходимость. Положим в $(3.19) \mathbf{v}=\left(1 \pm \varepsilon_{1}\right) \mathbf{u}, \varepsilon_{1}>0$, и перейдем к пределу при $\varepsilon_{1} \rightarrow 0$ :

$$
\int_{0}^{t} \int_{\Omega}\left(\overline{\mathbb{P}}+\overline{\mathbb{P}}_{b}\right): \mathbb{D}(\mathbf{u}) d \mathbf{x} d s=N(\mathbf{u}) .
$$


Такой предельный переход основан на аксиоме А4 для $\mathbb{P}$ :

$$
\mathbb{P}\left(\mathbf{u} \pm \varepsilon_{1} \mathbf{u}\right) \rightarrow \mathbb{P}(\mathbf{u}) \quad \text { *-слабо в } L_{\bar{M}}\left(Q_{T}\right),
$$

и непрерывности тензора $\mathbb{P}_{b 0}$ на лучах

$$
\mathbb{P}_{b 0}\left(\mathbf{u} \pm \varepsilon_{1} \mathbf{u}\right) \rightarrow \mathbb{P}_{b 0}(\mathbf{u}) \quad \text { п.в. }
$$

(и потому сильно в любом $\left.L_{p}\left(Q_{T}\right)\right)$. Сравнивая $(3.17)$ и $(3.20)$, используя представление $(1.7)$ и $*$-слабую полунепрерывность снизу функционала $\int_{\Omega} \varkappa(\mathbb{D}) d \mathbf{x}($ см. замечание 1.4), мы можем заключить $\int_{0}^{t} L(\mathbf{u}) d s \geqslant \varlimsup \lim \int_{0}^{t} L\left(\mathbf{u}_{\varepsilon}\right) d s$, что ввиду (3.9) и аксиомы $\mathrm{A} 7$ влечет сходимость $\mathbb{D}\left(\mathbf{u}_{\varepsilon_{k}}\right) \rightarrow \mathbb{D}(\mathbf{u})$ п.в. в $Q_{T}$, а потому (ввиду компактности по мере и равностепенной непрерывности норм в $L_{p}[11 ;$ с. 117, 135]) и сильно в любом $L_{p}\left(Q_{T}\right)$ с $p<+\infty$. Сравнивая теперь $(3.16)$ и $(3.20)$ и используя *-слабую полунепрерывность снизу функционала $N$ (см. замечание 1.4$)$, получаем $K(\rho) \geqslant \varlimsup \lim K\left(\rho_{\varepsilon_{k}}\right)$, что ввиду $(3.11)_{1}$ и примера 4.10 влечет сходимость $\rho_{\varepsilon_{k}} \rightarrow \rho$ п.в. в $Q_{T}$ и потому сильную в любом пространстве вида $L_{p}\left(0, T, L_{\Phi_{*}}(\Omega)\right)$ с $\Phi_{*} \prec \prec \Phi_{\beta}$ и $p<+\infty$ (аналогично). Кроме того, ввиду (3.20) соотношение (3.14) превращается B (3.4).

Этап V: "снятие черт" в (3.12). В силу аксиомы А5 для $\mathbb{P}$, оценки (3.6), сходимостей $\mathbb{D}\left(\mathbf{u}_{\varepsilon_{k}}\right) \rightarrow \mathbb{D}(\mathbf{u})$ п.в. в $Q_{T}$ и $(3.9)$ получаем $\mathbb{P}(\mathbf{u})=\overline{\mathbb{P}}$.

На множестве $F=\{\mathbb{D}(\mathbf{u}) \neq 0\}$ в силу свойства $\mathrm{C} 7$ имеем $\mathbb{P}_{b \varepsilon_{k}}\left(\mathbf{u}_{\varepsilon_{k}}\right) \rightarrow \mathbb{P}_{b}(\mathbf{u})$ п.в., а значит, и в норме $L_{p}(F)$ при всех $p<+\infty$ (благодаря C1). Значит, $\mathbb{P}_{b}(\mathbf{u})=\overline{\mathbb{P}}_{b}$ на $F$.

На $Q_{T} \backslash F$ мы можем лишь сказать, что (в силу C1) $\mathbb{P}_{b \varepsilon}\left(\mathbf{u}_{\varepsilon}\right) \in p_{*} \bar{P}$, а потому ввиду (3.9) и примера 4.5 с $K=p_{*} \overline{\mathscr{P}}$ также и

$$
\overline{\mathbb{P}}_{b}=\underset{L_{\infty}\left(Q_{T} \backslash F\right)}{*-w} \lim _{\varepsilon \rightarrow 0} \mathbb{P}_{b \varepsilon}\left(\mathbf{u}_{\varepsilon}\right) \in p_{*} \overline{\mathscr{P}} .
$$

Таким образом, на всем $Q_{T}$ имеем $\overline{\mathbb{P}}_{b}=\mathbb{P}_{b}(\mathbf{u})$ в смысле многозначной функции $(0.4)$ и, следовательно, (3.12) превращается в (0.2), что доказывает пп. 1-3 теоремы. Учитывая замечание 3.3, мы можем завершить доказательство.

4. Вспомогательные утверждения. В отличие от случая слабой сходимости, *-слабая полунепрерывность снизу для выпуклых функционалов требует отдельных комментариев; кроме того, нас интересует обратное свойство (вывод более сильной сходимости из сходимости значений функционала). При этом для нас важны прежде всего пространства функций (в том числе векторнозначных) и интегральные функционалы на них. Тензоры из $\mathbb{S}_{n}$ в данном контексте можно рассматривать как векторы размерности $n^{2}$.

Начнем с простого утверждения.

ПреДЛОЖЕНИЕ 4.1. Пусть $F$ - произволъный бункционал в банаховом пространстве $X$ со значениями в $\mathbb{R} \cup\{+\infty\}$, а $N$ - сопряженный $\kappa$ нему функционал в $X^{*}$, m.e.

$$
N(\mathbf{u})=\sup _{\mathbf{v} \in X}((\mathbf{u}, \mathbf{v})-F(\mathbf{v})) \quad \forall \mathbf{u} \in X^{*} .
$$

Тогда $N$ *-слабо полунепрерывен снизу в $X^{*}$ :

$$
N(\mathbf{u}) \leqslant \underline{\lim } N\left(\mathbf{u}_{k}\right) \quad \forall \mathbf{u}_{k} \rightarrow \mathbf{u} \quad \text { *-слабо в } X^{*} .
$$


ДокАЗАТЕЛЬСТво тривиально.

Для интегральных функционалов $N$ в пространствах функций применение предложения 4.1 требует изучения операции сопряжения для функций в конечномерном пространстве:

$$
f^{*}(\mathbf{u})=\sup _{\mathbf{v} \in \mathbb{R}^{n}}((\mathbf{u}, \mathbf{v})-f(\mathbf{v}))
$$

где

$f: \mathbb{R}^{n} \rightarrow \mathbb{R} \cup\{+\infty\}, \quad f \not \equiv+\infty, \quad f$ полунепрерывна снизу и выпукла.

Класс (4.2) совпадает с множеством собственных замкнутых выпуклых функций $[15 ;$ с. 41,68$]$, на этом классе операция (4.1) взаимно однозначна и инволютивна: $f^{* *}=f[15 ;$ с. 120]. Для гладких строго выпуклых $f(4.1)$ есть преобразование Лежандра [15; с. 272], а для N-функций (4.1) дает обычную двойственность $\overline{(\cdot)}$.

УтВеРЖДЕНИЕ 4.2. Пусть $f$ - функиия класса (4.2). Рассмотрим функционал

$$
F(\mathbf{v})=\int_{\Omega} f(\mathbf{v}(\mathbf{x})) d \Omega
$$

как функционал из $X$ в $\mathbb{R} \cup\{+\infty\}$, где $X=L_{1}$ или $X=E_{\Phi}$ с некоторой $N$-функиией $\Phi$ (функиии из $X$ векторнозначные). Тогда $F^{*}=N$, где

$$
N(\mathbf{u})=\int_{\Omega} f^{*}(\mathbf{u}(\mathbf{x})) d \Omega
$$

(функционал из $X^{*}$ в $\left.\mathbb{R} \cup\{+\infty\}\right)$.

ДокАзАтЕЛьство. По определению (4.1) неравенство $F^{*} \leqslant N$ тривиально. Покажем обратное неравенство. Из (4.1) следует

$$
\forall \mathbf{u} \in \mathbb{R}^{n} \quad \forall \varepsilon>0 \quad \exists \mathbf{w}_{\varepsilon}(\mathbf{u}) \in \mathbb{R}^{n}: \quad\left(\mathbf{u}, \mathbf{w}_{\varepsilon}(\mathbf{u})\right)-f\left(\mathbf{w}_{\varepsilon}(\mathbf{u})\right) \geqslant f^{*}(\mathbf{u})-\varepsilon .
$$

Пусть $\mathbf{u} \in X^{*}$ таково, что $N(\mathbf{u})<+\infty$. Положим $\mathbf{v}_{\varepsilon}=T_{a(\varepsilon)}\left(\mathbf{w}_{\varepsilon}(\mathbf{u})\right)$, где $T_{N}$ : $\mathbf{b} \mapsto \mathbf{b} \chi_{\{|\mathbf{b}| \leqslant N\}}-$ оператор срезки, а числа $a(\varepsilon)$ будут уточнены далее. Тогда $\mathbf{v}_{\varepsilon} \in$ $L_{\infty} \subset X$, так что

$$
\begin{aligned}
F^{*}(\mathbf{u}) & \geqslant\left(\mathbf{u}, \mathbf{v}_{\varepsilon}\right)-F\left(\mathbf{v}_{\varepsilon}\right) \\
& =\int_{\left\{\left|\mathbf{w}_{\varepsilon}(\mathbf{u})\right| \leqslant a(\varepsilon)\right\}}\left[\left(\mathbf{u}, \mathbf{w}_{\varepsilon}(\mathbf{u})\right)-f\left(\mathbf{w}_{\varepsilon}(\mathbf{u})\right)\right] d \Omega-f(0) \operatorname{mes}\left\{\left|\mathbf{w}_{\varepsilon}(\mathbf{u})\right|>a(\varepsilon)\right\} \\
& \geqslant N(\mathbf{u})-\varepsilon \operatorname{mes} \Omega-\int_{\left\{\left|\mathbf{w}_{\varepsilon}(\mathbf{u})\right|>a(\varepsilon)\right\}}\left[f^{*}(\mathbf{u})+f(0)\right] d \Omega,
\end{aligned}
$$

и требуемое получается, если $\operatorname{mes}\left\{\left|\mathbf{w}_{\varepsilon}(\mathbf{u})\right|>a(\varepsilon)\right\} \rightarrow 0$ при $\varepsilon \rightarrow 0$, чего можно добиться выбором $a(\varepsilon)$. В случае $N(\mathbf{u})=+\infty$ доказательство проводится аналогично.

СлеДСТвИЕ 4.3. Пусть $g$ - функиия класса (4.2). Тогда $f=g^{*}$ удовлетворяет условиям утверждения 4.2, а, значит, по предложению 4.1 бункиионал

$$
N(\mathbf{u})=\int_{\Omega} g(\mathbf{u}(\mathbf{x})) d \Omega
$$

*-слабо полунепрерывен снизу во всех пространствах Орлича и в $L_{\infty}$. 
ПРИМЕР 4.4. Пусть $n=1$,

$$
g(v)= \begin{cases}+\infty, & v<0 \\ v \ln v, & v \geqslant 0\end{cases}
$$

Ввиду следствия 4.3 получаем, что функционал $K(u)=\int_{\Omega} u \ln u d \Omega$, рассматриваемый на функциях $u \geqslant 0, *$-слабо полунепрерывен снизу во всех пространствах Орлича и в $L_{\infty}$.

ПримеР 4.5. Пусть $K$ - выпуклое замкнутое множество. Его индикаторная функция [15; с. 45]

$$
\delta(\mathbf{v} \mid K)= \begin{cases}0, & \mathbf{v} \in K \\ +\infty & \text { иначе }\end{cases}
$$

класса (4.2), поэтому функционал

$$
N(\mathbf{u})=\int_{\Omega} \delta(\mathbf{u} \mid K) d \Omega
$$

*-слабо полунепрерывен снизу на $L_{\infty}$. Это, в частности, означает, что если последовательность функций $\mathbf{u}_{k}$ принимает значения только в $K$ и сходится $*$-слабо в $L_{\infty}$, то предел также принимает значения только в $K$.

Перейдем к рассмотрению обратной ситуации, когда предел значений функционала равен его значению на *-слабом пределе, и мы пытаемся усилить сходимость. Как известно, для абстрактных пространств $X$ такого рода рассуждения требуют равномерной выпуклости (и, как следствие, рефлексивности) $X^{*}$, что не годится в нашей ситуации (пространства Орлича и $L_{\infty}$ не рефлексивны). Поэтому мы сразу ограничимся интегральными функционалами.

УтверЖДЕнИЕ 4.6. Пусть функиия $f: A \rightarrow \mathbb{R}$ класса $C^{2}(A)$, где $A \subset \mathbb{R}^{n}$ - область, причем тензор $D^{2} f$ вторых производных положителен, а точнее

$$
D^{2} f(\mathbf{s})\langle\xi, \xi\rangle \geqslant \frac{\gamma|\xi|^{2}}{1+|\mathbf{s}|^{2}} \quad \forall \mathbf{s} \in A, \quad \xi \in \mathbb{R}^{n},
$$

где $\gamma=$ const $>0$. Пусть $L_{\Phi}(\Omega)$ - пространство Орлича из функций со значениями в $\mathbb{R}^{n}, \mathbf{z}_{n} \rightarrow \mathbf{z}$ *-слабо в $L_{\Phi}(\Omega)$, причем все $\mathbf{z}_{n}$ и $\mathbf{z}$ принимают значения в $A, a$

$$
D f(\mathbf{z}) \in E_{\bar{\Phi}}(\Omega), \quad \underline{\lim } \int_{\Omega} f\left(\mathbf{z}_{n}\right) d \Omega \leqslant \int_{\Omega} f(\mathbf{z}) d \Omega .
$$

Тогда из $\left\{\mathbf{z}_{n}\right\}$ можно выделить подпоследовательность, сходяшуюся $\kappa \mathbf{z}$ п.в. в $\Omega$.

ДокАзАтЕЛЬСтво. Если $n=1, A=(\alpha, \beta),-\infty \leqslant \alpha<\beta \leqslant+\infty$, то для всех $x, y \in(\alpha, \beta), x \neq y$, определена величина

$$
g_{f}(x, y)=f^{\prime-1}\left(\frac{f(x)-f(y)}{x-y}\right),
$$

которую мы доопределим как $g_{f}(x, x)=x$, тогда $g_{f}(x, y)=g_{f}(y, x)$. Величина $g_{f}$ определена на $(\alpha, \beta)^{2}$ однозначно в силу строгой выпуклости $f$ она есть промежуточная точка в формуле конечных приращений $f(x)-f(y)=f^{\prime}\left(g_{f}(x, y)\right)(x-y)$. Легко проверить (на основании строгой выпуклости $f$ ), что

1) $g_{f}(x, y)$ лежит строго внутри отрезка $\langle x, y\rangle$ с концами в точках $x$ и $y$;

2) $g_{f}(\cdot, y)$ строго возрастает при всех $y \in(\alpha, \beta)$. 
При $n>1$ функция $f$ строго выпукла и класса $C^{2}$ на любом отрезке из $A$, так что снова можно ввести промежуточную точку $g_{f}(\mathbf{x}, \mathbf{y}) \in\langle\mathbf{x}, \mathbf{y}\rangle$ (но теперь в (4.5) следует понимать под $f^{\prime}$ производную по направлению $\mathbf{x}-\mathbf{y}$, а в знаменателе поставить $|\mathbf{x}-\mathbf{y}|)$, и она сохраняет свойства 1), 2) на отрезках. Формула конечных приращений принимает вид

$$
f(\mathbf{x})-f(\mathbf{y})=D f\left(g_{f}(\mathbf{x}, \mathbf{y})\right) \cdot(\mathbf{x}-\mathbf{y}) \quad \forall(\mathbf{x}, \mathbf{y}) \in A^{2} .
$$

Лемма 4.7. Если $\mathbf{x}_{n}, \mathbf{x} \in A, m o$

$$
g_{f}\left(\mathbf{x}_{n}, \mathbf{x}\right) \rightarrow \mathbf{x} \quad \Rightarrow \quad \mathbf{x}_{n} \rightarrow \mathbf{x}
$$

ДокАЗАТЕЛЬСтво. Пусть $\mathbf{x}_{n} \nrightarrow \mathbf{x}$, так что можно считать $\left|\mathbf{x}_{n}-\mathbf{x}\right| \geqslant \varepsilon_{0}=$ const $>0$. Обозначим

$$
\mathbf{x}_{n}^{\prime}=\mathbf{x}+\varepsilon_{0} \frac{\mathbf{x}_{n}-\mathbf{x}}{\left|\mathbf{x}_{n}-\mathbf{x}\right|} \in\left\langle\mathbf{x}_{n}, \mathbf{x}\right\rangle \cap S\left(\mathbf{x}, \varepsilon_{0}\right) .
$$

В силу свойства 2) имеем $\left|g_{f}\left(\mathbf{x}_{n}, \mathbf{x}\right)-g_{f}(\mathbf{x}, \mathbf{x})\right| \geqslant\left|g_{f}\left(\mathbf{x}_{n}^{\prime}, \mathbf{x}\right)-g_{f}(\mathbf{x}, \mathbf{x})\right|$, т.е.

$$
\left|g_{f}\left(\mathbf{x}_{n}, \mathbf{x}\right)-\mathbf{x}\right| \geqslant \inf _{\mathbf{y} \in S\left(\mathbf{x}, \varepsilon_{0}\right)}\left|g_{f}(\mathbf{y}, \mathbf{x})-\mathbf{x}\right|=\text { const }>0
$$

так как из $f \in C^{2}\left(B\left(\mathbf{x}, \varepsilon_{0}\right)\right)$ очевидно следует $g_{f}(\cdot, \mathbf{x}) \in C\left(S\left(\mathbf{x}, \varepsilon_{0}\right)\right)$, a $g_{f}(\cdot, \mathbf{x}) \neq \mathbf{x}$ на $S\left(\mathbf{x}, \varepsilon_{0}\right)$ в силу свойства 2$)$.

Лемма 4.8. Для всех $\mathbf{a}, \mathbf{b} \in A$ верно

$$
[D f(\mathbf{a})-D f(\mathbf{b})] \cdot[\mathbf{a}-\mathbf{b}] \geqslant \varphi(\mathbf{b}, \mathbf{a}-\mathbf{b}) \geqslant 0,
$$

где функиия ч обладает свойством

$$
\left[\exists \mathbf{y} \in A: \varphi\left(\mathbf{y}, \xi_{k}\right) \rightarrow 0\right] \quad \Rightarrow \quad\left[\xi_{k} \rightarrow 0\right] .
$$

ДоказАтельство. Для всех $\mathbf{a}, \mathbf{b} \in A$ имеем

$$
[D f(\mathbf{a})-D f(\mathbf{b})] \cdot[\mathbf{a}-\mathbf{b}]=\left(D^{2} f\right)(\mathbf{h})\langle\mathbf{a}-\mathbf{b}, \mathbf{a}-\mathbf{b}\rangle, \quad \mathbf{h} \in\langle\mathbf{a}, \mathbf{b}\rangle .
$$

В самом деле, поворотом системы координат можно добиться параллельности вектора $\mathbf{a}-\mathbf{b}$ одной из координатных осей, а тогда (4.10) очевидно. Легко понять, что $|\mathbf{h}| \leqslant|\mathbf{b}|+|\mathbf{a}-\mathbf{b}|$, поэтому (4.3) и (4.10) дают (4.8) с функцией

$$
\varphi(\mathbf{y}, \xi)=\frac{\gamma|\xi|^{2}}{1+(|\mathbf{y}|+|\xi|)^{2}},
$$

которая удовлетворяет $(4.9)$, так как $\varphi(\mathbf{y}, \cdot)$ непрерывна, равна 0 только в 0 , а на $\infty$ стремится к $\gamma>0$.

Теперь, используя (4.6), (4.8), свойство 2) и монотонность $D f$, мы можем написать цепочку неравенств для любых $\mathbf{x}, \mathbf{y} \in A$ :

$$
\begin{aligned}
{[f(\mathbf{x})-f(\mathbf{y})]-D f(\mathbf{y}) \cdot(\mathbf{x}-\mathbf{y}) } & \geqslant\left[D f\left(g_{f}(\mathbf{x}, \mathbf{y})\right)-D f(\mathbf{y})\right] \cdot\left[g_{f}(\mathbf{x}, \mathbf{y})-\mathbf{y}\right] \\
& \geqslant \varphi\left(\mathbf{y}, g_{f}(\mathbf{x}, \mathbf{y})-\mathbf{y}\right) \geqslant 0 .
\end{aligned}
$$

Полагая $\mathbf{x}=\mathbf{z}_{n}, \mathbf{y}=\mathbf{z}$ и интегрируя по $\Omega$, получим $\varphi\left(\mathbf{z}, g_{f}\left(\mathbf{z}_{n}, \mathbf{z}\right)-\mathbf{z}\right) \rightarrow 0$ в $L_{1}(\Omega)$, значит, для некоторой подпоследовательности эта сходимость имеет место п.в. в $\Omega$. Применяя теперь (4.9), а затем (4.7), получим требуемое. Утверждение 4.6 доказано. 
ЗАмечАниЕ 4.9. Рассмотрение случая $n=1$ показывает, что ограничение (4.3) нельзя ослабить, если требуется (4.9).

ПримеР 4.10. Пусть $n=1, A=\mathbb{R}^{+}, f(s)=s \ln s$. Тогда (4.3) выполнено с $\gamma=2$. Таким образом, мы попадаем в условия утверждения 4.6, если $z_{n}, z \in L_{\Phi}, \ln z \in E_{\bar{\Phi}}$, т.е. $\bar{\Phi}(C \ln z) \in L_{1}$ для всех $C>0$. Легко проверить, что при $\Phi=\Phi_{\beta}, \beta>1$, из $z \in L_{\Phi}$ вытекает $\ln z \in E_{\bar{\Phi}}$, т.е. (4.4) 1 выполнено автоматически.

ПримеР 4.11. Пусть $A=\mathbb{S}_{n}, f_{s}(\mathbb{D})=\Gamma_{s}^{\prime}\left(\left|\mathbb{D}^{s}\right|^{2}\right)\left|\mathbb{D}^{s}\right|^{2}$ или $f_{0}(\mathbb{D})=\Lambda^{\prime}\left((\operatorname{tr} \mathbb{D})^{2}\right)(\operatorname{tr} \mathbb{D})^{2}$ из таких слагаемых составлен функционал $L$ в примере $1.1 ; \Phi=M ; \mathbf{z}:=\mathbb{D}(\mathbf{u})$, $\mathbf{z}_{\varepsilon}:=\mathbb{D}\left(\mathbf{u}_{\varepsilon}\right)$. Условия утверждения 4.6 сводятся к требованиям строгой выпуклости функций $f_{s}$ (а точнее, оценкам вида $\left.(4.3)\right)$ и достаточной гладкости.

\section{СПИСОК ЦИТИРОВАННОЙ ЛИТЕРАТУРЫ}

[1] E. C. Bingham, Fluidity and Plasticity, McGrew-Hill Book Co., New York, 1922.

[2] W. Prager, Introduction to Mechanics of Continua, Introductions to Higher Mathematics, Ginn and Co., New York, 1961.

[3] Дж. Серрин, Математические основы классической механики жидкости, ИЛ, М., 1963.

[4] D. Prasad, H. K. Kytomaa, "Particle stress and viscous compaction during shear of dense suspensions", Intern. J. of Multiphase Flow, 21:5 (1995), 775-785.

[5] L. E. Malvern, Introduction to the Mechanics of a Continuous Medium, Prentice-Hall, Inc. Englewood Cliffs, New Jersey, 1969.

[6] I. V. Basov, V. V. Shelukhin, "Generalized solutions to the equations of compressible Bingham flows", ZAMM Z. Angew. Math. Mech., 79:3 (1999), 185-192.

[7] V. V. Shelukhin, "Bingham viscoplastic as a limit of non-newtonian fluids", J. Math. Fluid Mech., 4:2 (2002), 109-127.

[8] J. Málek, M. Růžička, V. V. Shelukhin, "Hershel-Bulkley fluids: existence and regularity of steady flows", Math. Models Methods Appl. Sci., 15:12 (2005), 1845-1861.

[9] А. Е. Мамонтов, "О глобальной разрешимости многомерных уравнений Навье-Стокса сжимаемой нелинейно вязкой жидкости. I”, Сиб. матем. журн., 40:2 (1999), 408-420.

[10] А. Е. Мамонтов, "О глобальной разрешимости многомерных уравнений Навье-Стокса сжимаемой нелинейно вязкой жидкости. II”, Сиб. матем. журн., 40:3 (1999), 635-649.

[11] М. А. Красносельский, Я. Б. Рутицкий, Выпуклье функиии и пространства Орлича, Современные проблемы математики, Физматгиз, М., 1958.

[12] А. Е. Мамонтов, "Оценки глобальной регулярности для многомерных уравнений сжимаемой неньютоновской жидкости", Матем. заметки, 68:3 (2000), 360-376.

[13] А. В. Кажихов, А. Е. Мамонтов, "Об одном классе выпуклых функций и точных классах корректности задачи Коши для уравнения переноса в пространствах Орлича", Сиб. матем. журн., 39:4 (1998), 831-850.

[14] Ж.-Л. Лионс, Некоторые методы решения нелинейных краевых задач, Мир, М., 1972.

[15] Р. Рокафеллар, Выпуклый анализ, Мир, М., 1973.

А. Е. Мамонтов

Институт гидродинамики им. М. А. Лаврентьева СО РАН, г. Новосибирск

E-mail: relic@hydro.nsc.ru
Поступило

24.11.2006

Исправленный вариант

11.04.2007 